\title{
JÜRGEN HABERMAS: TEORÍA DE LA ACCIÓN COMUNICATIVA. ACCIÓN Y RACIONALIDAD COMUNICATIVA EN EL MARCO DE LA TEORÍA CRÍTICA
}

\author{
KeYLOR VÁsQuez
}

La presente investigación es un desarrollo parcial -y considero que en muchos aspectos incompleto- que tiene por objetivo aclarar algunos conceptos básicos de la monumental obra de Jürgen Habermas, Teoría de la acción comunicativa. Dicho esto, el trabajo que presentaré no pretende ser una exposición sistemática del pensamiento habermasiano, sino, en primer lugar, una indagación sobre un problema conceptual determinado: qué responde Habermas a la tríada de preguntas iqué es acción comunicativa?, ¿cuál es su composición? y icuál es su finalidad? Planteado el primer problema de esta manera, se podrá evidenciar más adelante que aquello a lo que Habermas llama acción comunicativa, en principio, está orientado hacia el entendimiento y comprensión entre individuos $y$, por lo tanto, se distingue de otros tipos de acción orientados hacia fines. En segundo lugar, se intentará brindar una aproximación al lector sobre aquello que Habermas denomina racionalidad comunicativa. Por un lado, este tipo de racionalidad es presentada por Habermas en oposición a los tipos de racionalidad "instrumental" y "estratégica". Por otro lado, Habermas entiende ambas formas de racionalidad como ejes centrales de la tesis que le permite a Max Weber criticar los mecanismos de dominación de la acción social. De ahí que Habermas considere necesaria la introducción de la racionalidad comunicativa a fin de complementar y hacer viable la posibilidad de plantear una crítica original de la sociedad moderna en las extensas páginas de su obra Teoría de la acción comunicativa. Finalmente, como únicas tentativas, sí pretendo otorgar al lector, en cierta medida, una reflexión original sobre la teoría del actuar comunicativo y sus alcances en el 
universo de procesos sociales de la modernidad. Asimismo, pretendo realizar una mediana exploración de la actualidad de la Teoría Critica bajo la óptica de esta magna obra de Jürgen Habermas a través de la siguiente pregunta: hoy en día, ¿cuál es la vigencia y qué alcances puede tener una eventual crítica inmanente a la sociedad?

El primer filósofo en considerar que la época moderna portaba sobre sí un germen problemático fue Hegel. En cierto modo, se podría decir que Hegel avizoró las consecuencias políticas y filosóficas que llegarían una vez que el antiguo régimen cediera el paso a la modernidad'. La modernidad, más allá de perfilar un nuevo horizonte donde el espíritu pueda desplegarse y objetivar su razón en un sujeto histórico, concebía en su seno el nuevo paradigma de las filosofías modernas, a saber, la filosofía de la conciencia. Subjetividad y razón se convertirían, así, en las herramientas inapelables de la investigación y del saber. La pretensión del saber llevó al sujeto histórico, al hombre, a emprender la titánica búsqueda de encontrar un primer fundamento para la objetividad de su saber particular y de la racionalidad impuesta sobre el mundo que lo rodeaba. Hoy en día parece que aquella pretensión tiene aún efectos residuales en las ciencias sociales y en los mecanismos de dominación de no pocas sociedades. La ciencia y la técnica, herramientas poderosas desarrolladas por el hombre moderno, a decir de Adela Cortina, "realizan la tarea de dar un barniz de racionalidad al dominio del hombre por el hombre de la naturaleza por el hombre"2. Por ello, el aspecto crítico y gnoseológico donde la Teoría Crítica tuvo lugar de nacimiento, lejos de encontrarse realizado y consumado, sigue en constante transformación y actualización. Sobre esto último, siguiendo a Habermas, la modernidad es un proyecto inacabado y, como tal, las bases normativas de la crítica social tienen todavía vigencia conceptual que, a su vez, validan y hacen posible evocar objetivos por definirse.

La escuela de Frankfurt se sitúa como una institución heredera del pensamiento hegeliano-marxista ${ }^{3}$. Su origen procede de investigaciones sociales surgidas

I Cf. Habermas, Jürgen, El discurso filosófico de la modernidad, Buenos Aires: Katz, 2008, p. 26. Sobre Hegel, el concepto de la modernidad y las consecuencias de la misma, véase también la sección "Hegel: concepto de modernidad" de la mencionada obra.

2 Cortina, Adela, La escuela de Fráncfort. Crítica y utopía, Madrid: Síntesis, 2008, p. 94. 
para efectos de estudios marxistas organizados por Félix Weil en 1922. En su libro Historia y conciencia de clase ${ }^{4}$, Lukács realiza un primer -o uno de los primeros-análisis tomando por marco de interpretación las bases dialécticas del joven Marx acerca del proceso de cosificación de la conciencia. A partir de este método dialéctico de análisis de los procesos de producción de vida del hombre en la sociedad, planteó una revolución autorreflexiva que combata los mecanismos burgueses de dominación. Tiempo después, hacia 193I, Max Horkheimer asumirá la dirección de la Escuela y, por tanto, de su orientación científica-social, acentuando la reflexión filosófica como una necesidad imperante ante las ortodoxias marxistas de la época. En este contexto se va gestando el proyecto de lo que hoy llamamos Teoría Crítica.

De un modo u otro, para Horkheimer era momento de cambiar la dirección filosófica, de enlazar una propuesta revolucionaria marxista con un proyecto filosófico, con el objetivo de liberar la conciencia del hombre hacia el horizonte de sus propias finalidades prácticas. La posición teórica de Max Horkheimer, se podría decir, hace hincapié de la tesis de Max Weber sobre el uso de los conceptos de racionalización y crítica instrumental. Hasta aquí en lo que respecta a la tradición Marx-Lukács. Posteriormente, Horkheimer, en compañía de Adorno y en el exilio de los años cuarenta, escribe la Dialéctica de la llustración con la intención de construir una sólida autocrítica radical que haga frente a la razón instrumental planteada por Lukács. En el exilio, ambos conceptualizan la sociedad y el sujeto en términos de control racional y dominación técnica de la naturaleza desmitologizada ${ }^{5}$. La dominación en la sociedad capitalista, a decir de Horkheimer y Adorno, queda institucionalizada en sus dos manifestaciones. La razón instrumental que, en principio, se mostraba como dominio de una naturaleza externa, escondía en su seno el dominio de la naturaleza interna del individuo, de su subjetividad. Al cabo de se proceso los individuos son despojados de su propia naturaleza en la misma medida que la sociedad capitalista, cada vez más administrada respecto de fines, encarna la razón instrumental ${ }^{6}$.

${ }^{4}$ Cf. Lukács, György, Historia y conciencia de clase, México D.F.: Grijalbo, 1969.

5 Cf. Horkheimer, Max y Theodor Adorno, Dialéctica de la llustración, Madrid: Trotta, 1998. El tema de la racionalización, dominación de la naturaleza y desmitologización se encuentran fundamentalmente en la sección titulada "Concepto de llustración" de la citada obra.

6 Cf. Habermas, Jürgen, Teoría de la acción comunicativa: complementos y estudios previos, Madrid: Cátedra, 200I, p. 378. 
Habermas y su teoría de la acción comunicativa presuponen, en primer lugar, las condiciones de socialización moderna. La teoría habermasiana desarrolla la tesis weberiana allí donde toda acción del individuo está en función de los fines de una organización o sistema que rige a partir de acciones instrumentales y estratégicas. Ante este tratamiento que ocupa gran importancia en la producción crítica de Habermas, se plantea el concepto de acción comunicativa para ampliar el espectro del significado de acción. Este innovador planteamiento describe a individuos que hacen uso preferente de la racionalidad comunicativa, en principio, no para conseguir ventajas respecto de fines propios, sino, más bien, para el entendimiento. De esta forma, Habermas procede a identificar cada caso mediante el uso de descripciones distintas según el modelo. Es decir, por un lado, una acción, en sentido estricto, es una acción no lingüística que tiende, en las actividades teleológicas, a la consecución de un fin preconcebido por el actor en el mundo; mientras que, por otro lado, las expresiones lingüísticas son descritas como acciones que, lejos de perseguir algún interés o éxito personales, se orientan a la comprensión y entendimiento mutuo de algo acerca del mundo. Este análisis de Habermas se encuentra bajo la perspectiva del agente, desde la óptica de la primera persona. En cambio, la descripción hecha en la óptica de una tercera persona será, pues, la de "cómo un actor (agente) por medio de una actividad teleológica consigue un fin o cómo mediante un acto de habla se entiende con alguien acerca de algo"7.

Retomando la argumentación en torno a la acción comunicativa, este tipo de acción posee, como componente esencial, la coordinación de la acción en el uso del lenguaje. El acto de comunicación eleva pretensiones de validez implícitas en nuestra experiencia cotidiana de socialización y las lleva hacia la comprensión, porque en este tipo de acción yacen presupuestos tales como competencia comunicativa, situación ideal de habla, consenso, base de validez del habla, etc. Con tales presupuestos contenidos en el fondo de teoría del actuar comunicativo, la búsqueda de Habermas de identificar los conceptos a priori para analizar las estructuras generales del actuar orientado a la comprensión se halla suficientemente completada. En ese sentido, Axel Honneth considera que "[Habermas] inicialmente realiza su teoría de la acción bajo la forma de una pragmática universal (...) [donde] su objetivo específico consiste 
en demostrar que, en el ámbito del discurso orientado al entendimiento, los sujetos interactúan entre si”"

Considero oportuno resaltar de lo dicho líneas arriba que aquellos individuos presentados por Habermas están bajo condiciones simétricas regidas por una base de validez normativa que eleva sus pretensiones de habla y que en "esta práctica de la acción comunicativa se introducen parámetros universales de racionalización que poseen validez obligatoria al margen de la conciencia"’. En consecuencia, a partir de lo dicho por Honneth, se amplía el espectro de racionalidad social weberiano que obliga a otros ámbitos de la existencia ${ }^{10} \mathrm{a}$ acomodarse a las formas de racionalidad instrumental y racionalidad estratégica. Para Habermas, la triada de ámbitos señalados por Weber conformaría un único ámbito conceptual (fenomenológico) denominado mundo de la vida.

La racionalidad estratégica es propia de los individuos en la medida en que la racionalidad instrumental lo es respecto de fines. Pero, ¿qué relación guardan los aspectos de la racionalidad de la acción estratégica con la racionalización social? En el mundo de la vida no es la racionalidad de los individuos la que prima en las estructuras socioeconómicas sino, desde el funcionalismo sociológico, la racionalidad sistémica, entendiéndose ésta como la racionalidad instrumental con arreglo a fines cuyo mantenimiento de las estructuras sistémicas se convierte en el fin supremo del individuo. La clave de la preservación de estas estructuras sistémicas se halla en la desaparición del saber y la racionalidad de la acción de quienes conforman el corpus orgánico del sistema, los individuos. Sin embargo, en el momento en que Weber introduce el concepto de subsistemas, donde ubica a la economía, la política, el derecho, etc., los procesos de racionalización social retoman la problemática del saber de los individuos no tomados en cuenta por el funcionalismo sociológico, ya que los

8 Honneth, Axel, “La teoría de la sociedad de Habermas", en: Crítica del poder:fases en la reflexión de una teoría crítica de la sociedad, Madrid: A. Machado Libros, 2009, p. 4I4. Los corchetes son míos.

9 Ibid.

10 Para Weber, estas otras existencias vendrían a ser los ámbitos de la naturaleza, de la vida y de lo social. 
subsistemas cargan consigo medios de relación -parcial o absoluta- entre subsistema e individuos. Estos medios de relación son el dinero y el poder".

En general, a la luz del desarrollo planteado en Teoría de la acción comunicativa, el concepto de racionalidad comunicativa debe entenderse en oposición al de racionalidad respecto de fines de Max Weber. Habermas centra su análisis en el concepto de racionalización de Weber porque éste lo contempla como las relaciones de los sujetos con el mundo, como si las acciones producidas por el binomio sujeto-mundo tuvieran un aspecto inherente que tiende a la consecución de fines sistémicos. El análisis de Habermas permite desmitologizar la aparente generalidad de la racionalización weberiana, tomada por imperante y universalmente válida en toda sociedad capitalista. Con todo, Habermas sostendrá que el concepto de racionalización comunicativa hasta aquí expuesto, en primer lugar, cumple la función de alcanzar metas (entendimiento) y, en segundo lugar, constituye en su ser un saber empírico (como procesos de relaciones sociales). En otras palabras, para Habermas, racionalización no es otra cosa que el uso comunicativo constituyente de la experiencia humana cuyas acciones llevan finalmente a un consenso.

En el acto comunicativo, "los distintos participantes superan sus opiniones inicialmente subjetivas y aseguran, gracias al compartimiento de convicciones racionalmente motivadas, al mismo tiempo la unidad del mundo objetivo y la intersubjetividad de su contexto de vida"'2 . La oposición teórica entre acciones con arreglo a fines y acciones comunicativas es forzosa. Aun cuando no tenga intención de realizar un tratamiento exhaustivo de cada una de ellas en el presente trabajo, no desmerecería relevancia alguna hacerlo. Sin embargo, debo acentuar la importancia del actuar comunicativo porque éste se da en el ámbito práctico de la vida cotidiana; mientras que, por otro lado, la acción racional con arreglo a fines "parte del actor que se orienta exclusivamente por la consecución de su objetivo global conforme a fines concretos y somete

"Cf. Habermas, Jürgen, Teoría de la acción comunicativa: complementos y estudios previos, p. 376. Asimismo, sobre el dinero y el poder como medios de relación y "coordinación de esas acciones racionales conforme a fines que contribuyen al dominio de la reproducción material evitando pagar el coste de toda comunicación lingüística" cercenada para todo fin del funcionalismo sociológico, véase Honneth, Axel, op. cit., pp. 433-434.

12 Habermas, Jürgen. Teoría de la acción comunicativa: complementos y estudios previos, p. 28. 
a cálculo todas las demás consecuencias de la acción como condiciones secundarias de éxito al que aspira"'3.

Acción social, en Habermas, es conceptualmente equiparable a interacción social en Weber. La acción social, en tanto virtud del significado subjetivo depositada por el individuo como actor comunicativo, toma en consideración la manera como se desarrolla el comportamiento individual frente a la colectividad de su género. De ese modo, su acción queda orientada en el transcurso de esa interacción. La interacción viene a ser para Habermas una acción social orientada por la acción, la cual corresponde a una coordinación de la acción según intereses y acuerdos normativos.

Con la Teoría de la acción comunicativa, nuestro autor persigue el objetivo de elaborar un concepto de racionalización social que sea tan comprensivo como para posibilitar una crítica. Con ello, Habermas tendrá no solamente que oponerse al análisis marxista, sino también al concepto de racionalización weberiano y al diagnóstico de Horkheimer y Adorno acerca de la historia ${ }^{14}$. Intentará traer de vuelta las aspiraciones con las que se originó el proyecto de Teoría Crítica con el objetivo de alcanzar una posición mejor fundamentada y comprensiva de esas formas de socialización racional y unilateral conforme a fines ${ }^{15}$. Por ello, Habermas se verá forzado a dar un viraje teórico-comunicativo al diagnóstico filosófico-histórico ya planteado en la Dialéctica de la llustración.

En las relaciones sociales, el entendimiento parecer ser, a decir de Habermas, inmanente como telos del lenguaje. Por otra parte, no toda relación social que es establecida por medios lingüísticos es un ejemplo de acción comunicativa. Luego, la contradicción entre trabajo e interacción, entre razón instrumentaly razón comunicativa, tiene que ser superada en un análisis sistemático en el que por racional se defina exclusivamente una perspectiva inmanente a la acción comunicativa ${ }^{16}$. De ella se deducen tres formas de racionalidad inherentes al entendimiento lingüístico, toda vez que un locutor no solo busca elevar una

\footnotetext{
13 Ibid., p. 384.

${ }^{14}$ Cf. Honneth, Axel, op. cit., p. 419.

${ }^{15}$ Cf. ibid., p. 419 ss.

${ }^{16}$ Cf. Habermas, Jürgen, Teoría de la acción comunicativa: complementos y estudios previos, pp. I55, 372 ss.
} 
pretensión de verdad al proferir sus enunciados, sino que también supone corrección y veracidad de sus expresiones. Hasta aquí debe entenderse que una verdad corresponde a un hecho existente en el mundo objetivo, la corrección a la que alude se trata de seguir las normas morales del mundo social y, finalmente, su veracidad radica en el ámbito del mundo de la vida interna al sujeto. Así, yace la posibilidad de racionalización de la acción humana en tres dimensiones o tres referencias al mundo que coincide con la teoría de los tres mundos de Popper en dos postulados. El primer postulado señala que los componentes últimos del mundo social son individuos que actúan adecuadamente, de un modo u otro, a la luz de sus disposiciones y de la comprensión de su situación. El segundo postulado dice que si los individuos poseyeran una adecuada y competente información de la existente tendencia social, habría, por ende, alguna forma de modificarla a partir del saber constituyente que yace en los individuos provistos de lenguaje, haciéndolos las únicas fuerzas motoras en la evolución histórica de los sistemas sociales ${ }^{17}$. Para Honneth, "nosotros podemos, por consiguiente, incrementar nuestro conocimiento no sólo acerca del entorno físico [mundo objetivo], sino también del mundo de las normas sociales [mundo social] y de las experiencias subjetivas bajo la forma de procesos de aprendizaje, $y$, en esa medida, seguir desarrollando progresivamente la racionalidad de nuestra acción [mundo subjetivo]”|8.

El desplazamiento teórico que sucede al paradigma subjetivo toma por nombre paradigma de la intersubjetividad, donde lo que prevalece es la interacción en un proceso cooperativo de interpretación, resaltando en sus expresiones temáticamente uno de los tres componentes ya mencionados. Lo que interesa es el telos: comunicación y entendimiento. Entonces, a partir de lo que nos interesa, sería legítimo perfilar la racionalidad comunicativa como solución de los problemas que derivan de la cosificación de las relaciones comunicativas y, en última instancia, como posibilidad remota de los problemas prácticos de la vida cotidiana. Sin embargo, Habermas menciona que el actuar comunicativo es condición de posibilidad de una comunicación simétrica envuelta por la atmósfera de la racionalidad comunicativa, pero que la sociedad capitalista moderna lo recorta cada vez más a causa de patologías provenientes de las 
bases del mundo de la vida; es decir, el actuar comunicativo se vuelve un instrumento guiado por medios como el poder o el dinero. En consecuencia, estos medios impropios no solo ocasionan inexorablemente la sustitución del lenguaje como medio apropiado, sino que además establecen una función de coordinación (del actuar comunicativo) en función del poder o dinero ${ }^{19}$.

Lo anterior es un intento de alusión a una praxis específica de la acción comunicativa como un acto de entendimiento lingüístico en el que los participantes se ponen de acuerdo acerca de una interpretación común sobre la base de la validez del habla. En principio, se puede avizorar que en virtud de una coordinación de la acción social que conlleve a consenso (un entendimiento generalizado), resulta forzoso el abandono de la naturaleza interna en beneficio de la intersubjetividad. Ésta, como forma particular de coordinación de acciones dirigidas hacia su nuevo objetivo -el consenso-, construye procesos cooperativos de interpretación que no se agotan en el acto de entendimiento efectuado por la interpretación misma. Es decir, el telos no se limita, en el entendimiento y la cooperación, como producto de esta intersubjetividad, sino que además incorpora un proceso de coordinación de acción en las relaciones físicas, psíquicas, morales y cognitivas de un sujeto con su entorno práctico. De ahí que la posibilidad de establecer una teoría crítica de la sociedad se nos presente eventualmente como una patología de nuestra sociedad contemporánea, a consecuencia de la mutilación de estas considerables relaciones incorporadas al proceso de coordinación de acción del sujeto comunicativo que tiene por génesis el dualismo mundo de la vida y sistema. En vista de que las formas sistémicas de control amenazan con violentar y usurpar la dimensión de la praxis comunicativa, el autor de Teoría de la acción comunicativa señala que "los fenómenos específicos de alienación de las sociedades modernas se explican porque ámbitos del mundo de la vida comunicativamente estructurados quedan crecientemente sometidos a los imperativos de sistemas autonomizados que, por su parte, se diferenciaron a través de los medios dinero y poder, y que representan un fragmento de 'socialidad' exento de contenido normativo. Que el mundo de la vida dependa de 'integración social' y no pueda hacerse depender de mecanismos de

19 Cf. Habermas, Jürgen, Teoría de la acción comunicativa: complementos y estudios previos, p. 542. 
'integración sistémica' sin que se produzcan efectos cosificadores, necesita una explicación en términos de teoría de la comunicación" ${ }^{20}$.

Históricamente, el componente social se escinde, por un lado, en lo subjetivo, lo interno, lo comunicativo orientado a acciones de entendimiento; por otro lado, la separación origina lo objetivo, lo externo, lo instrumental respecto a fines. ¿Cómo entender entonces que, "si en realidad es la acción orientada al entendimiento, dentro de esas esferas diferenciadas de comunicación, la que exclusivamente cuida de la coordinación de los planes y proyectos de la acción social, quedan ya excluidos conceptualmente todos los posibles intentos de imposición de interés orientados al éxito" 21 ? Para dar cuenta de lo mencionado líneas arriba, haría falta un tratamiento serio y cuidadoso de los términos acuerdo e influencia que, por lo menos, me limitaré a señalar, más no a desarrollar. En Teoría de la acción comunicativa, ambos términos, si bien no son desarrollados ampliamente, son en buena cuenta señalados como mecanismos que se excluyen entre sí, entre coordinación lingüística de la acción social y coordinación no lingüística en el plano de integración social bajo la forma, por ejemplo, de ejercicio del poder.

A manera de conclusión, quisiera traer a colación la pregunta que a inicios de la presente exposición refirió a la actualidad de la Teoría Crítica y, más precisamente, a la relevancia actual de una crítica inmanente de la sociedad. A lo largo del corpus argumentativo, he intentado plantear que la teoría de la acción comunicativa tiene como tesis de fondo entender al lenguaje, $y$ al uso que hacemos de él, como un mecanismo originario de integración social. Además, adviértase que esta teoría refleja, de una u otra manera, hablantes que se reconocen mutua y necesariamente como interlocutores válidos, descubriendo en la performance de la acción comunicativa un reconocimiento básico que establece vínculos de sociabilización. De ahí que se pueda entender cierta fuente de obligación a través del reconocimiento recíproco de interlocutores que se saben, el uno para consigo y con el otro, imprescindibles para averiguar si la norma es o no justa. Este vínculo no es otra cosa que el entendimiento como medio de coordinación de la acción comunicativa. El concepto visto y 
tratado de acción comunicativa está pensado de tal manera que los actos del entendimiento que vinculan los planes de acción de múltiples participantes y que resumen las acciones orientadas a un objetivo en relación interactiva no pueden retrotraerse, por su parte, a una acción teleológica.

Habermas señala que "los procesos de entendimiento buscan un acuerdo que dependa de la aprobación racionalmente motivada al contenido de una aseveración. No es posible imponer el acuerdo a la otra parte ni se le puede imponer al interlocutor una manipulación"22. En tales condiciones, el reconocimiento recíproco es un vínculo que asegura la conexión, digamos, entre la autorreflexión o la conciencia de sí mismo y la orientación hacia el otro. Esta condición de relación con el otro, para Habermas, yace desde el origen en el reconocimiento de la alteridad (de ser el otro frente a uno) para poder reconocerme a mí mismo. El reconocimiento de este proceso se da en un espacio del mundo, en un contexto sociocultural y político determinado en el que el llamado mundo de la vida ofrece un conjunto de evidencias culturales que los participantes o actores hacen uso a lo largo de sus procesos de sociabilización en pro de sus esfuerzos interpretativos. Pues bien, el concepto de racionalización social propuesto por Habermas también se perfila sumándose al esfuerzo interpretativo y comprensivo a fin de posibilitar un crítica inmanente de la sociedad. Del mismo modo lo entiende Adela Cortina cuando señala que “(...) en el caso de la Escuela de Fráncfort, la crítica se sabe situada en unas determinadas condiciones socioeconómicas y, por tanto, no neutral, pero confía en la posibilidad de alcanzar la verdad objetiva sin conformarse con el perspectivismo de la sociología del conocimiento"23.

Precisamente, para hallar una posibilidad de crítica inmanente se presupone desmantelar los gigantes conceptos metafísicos que, contrariamente, ocultan la tarea de la crítica. Ésta parece consistir en sacar a la luz lo reprimido en la historia, puesto que un momento o una instancia de la historia es resultado de muchos otros que le anteceden, $y$ en desenmascarar las patologías sociales mediante algún criterio no metafísico, mas sí normativo. La reflexión nos revela que las cuestiones prácticas no son susceptibles de verdad o falsedad,

${ }^{22}$ Cf. Habermas, Jürgen, Conciencia moral y acción comunicativa, Barcelona: Península, I99I, p. 158.

${ }^{23}$ Cortina, Adela, op. cit., p. 145. 
pero sí de corrección o incorrección, pues nos regimos bajo ciertas normas y éstas, en la vida cotidiana, desempeñan una función al ser acatadas porque creemos tener razones para adoptarlas y cumplirlas sea cual fuera la magnitud de nuestras creencias razonables ${ }^{24}$.

Criterios de normatividad encontramos en la filosofía de la religión, en la teoría de la acción comunicativa o en cualquier otra teoría de evolución social. En efecto, siempre que la crítica se sepa, a sí misma, impulsada y guiada por un interés emancipatorio, la teoría crítica de la sociedad moderna actúa por disconformidad con la injusticia allí donde las condiciones de vida hacen imposible, por lo menos, formas de vida dignas para el hombre, aquel zoon politikon considerado por Aristóteles únicamente capaz de desarrollarse íntegramente en comunidad con los demás. Esta injusticia en las comunidades políticas modernas es el elemento central, recurrente e indisoluble de la Teoría Crítica, consciente de su inmersión en una realidad cambiante. Precisamente, a lo largo de la presente exposición, hemos sostenido que la meta de ésta consiste en diseñar una teoría social normativa y el tesón por descubrir y responder las demandas propias de un criterio para distinguir entre lo vigente y lo válido resulta, por tanto, esencial en ella ${ }^{25}$. 